\title{
Aplicación de la carta EWMA con base en la varianza efectiva para monitorear variabilidad en procesos de control de calidad multivariada de fungicidas
}

Application of the EWMA chart based on the effective variance to monitor variability in fungicides multivariate quality control processes

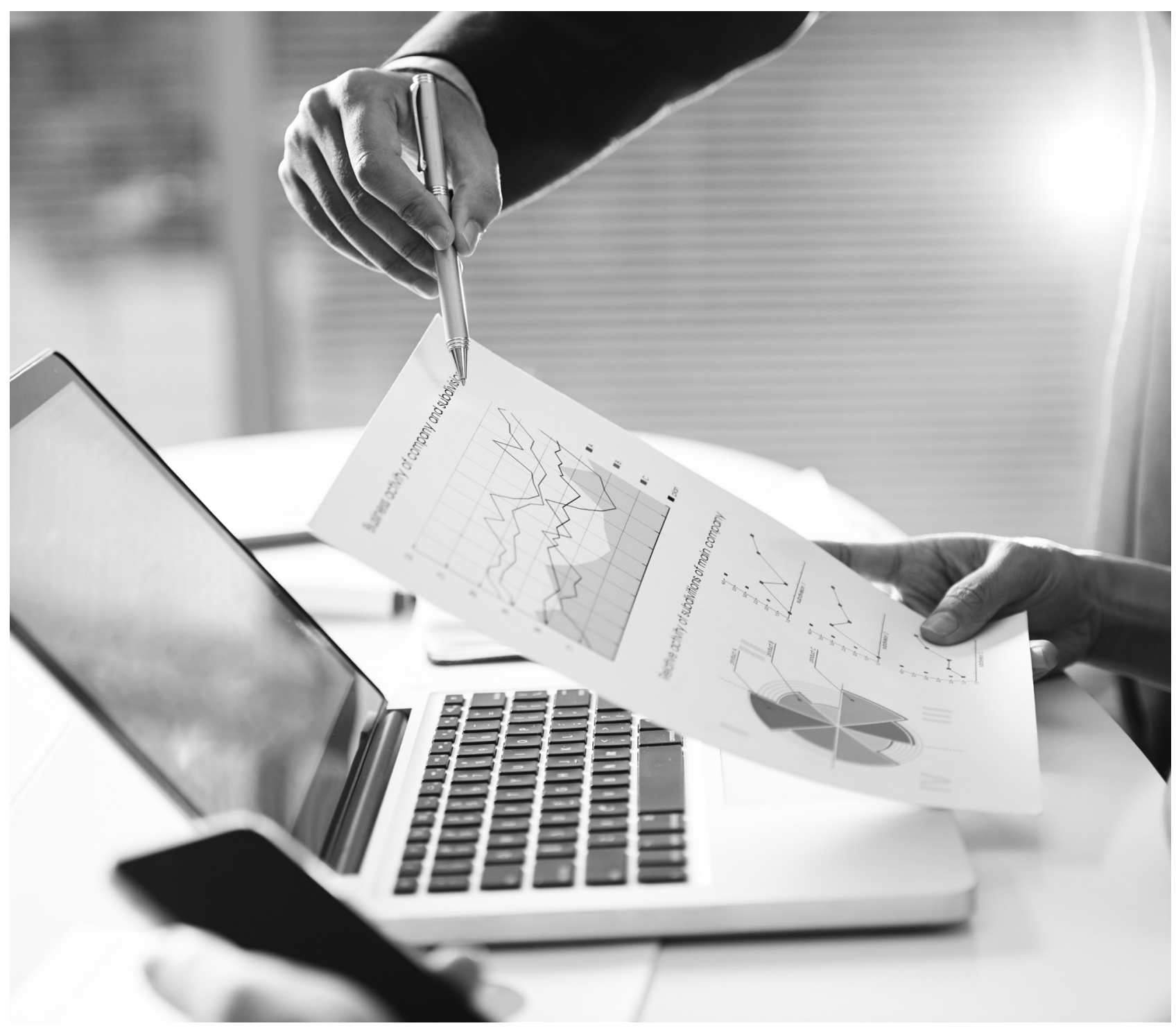




\title{
Aplicación de la carta EWMA con base en la varianza efectiva para monitorear variabilidad en procesos de control de calidad multivariada de fungicidas ${ }^{1}$
}

Application of the EWMA chart based on the effective variance to monitor variability in fungicides multivariate quality control processes

\section{Rogelio José Herrera-Chamorro ${ }^{2}$, Roberto José Herrera-Acosta ${ }^{3}$}

Artículo recibido en diciembre 6 de 2017; artículo aceptado en diciembre 11 de 2018.

\begin{abstract}
Este artículo puede compartirse bajo la Licencia Creative Commons Atribución-NoComercial-Compartirlgual 4.0 Internacional y se referencia usando el siguiente formato: Herrera-Chamorro, R. J. \& Herrera-Acosta, R. J. (2019). Aplicación de la carta EWMA con base en la varianza efectiva para monitorear variabilidad en procesos de control de calidad multivariada de fungicidas.

I+D Revista de Investigaciones, 14 (2), 33-39. DOI: https://doi.org/10.33304/revinv.v14n2-2019003
\end{abstract}

\section{Resumen}

En este artículo se presenta un caso de estudio en el que se realiza un monitoreo, mediante la carta de control EWMA con base en varianza efectiva. Esto permite evidenciar cómo el aumento de la variabilidad de una de las características de calidad afecta en forma sensible los resultados para este tipo de cartas. Adicionalmente, se propone aquí ampliar de esta manera la capacidad de selección de diferentes métodos de monitoreo de procesos multivariados. En este estudio se evalúan subgrupos sometidos en línea a un proceso de fabricación de protectores de planta o fungicidas, mediante dos variables resultantes del proceso, una vez establecidos los parámetros. Al analizar la carta de control EWMA con base en varianza efectiva, se encuentra que esta genera límites muy cerrados, lo que podría ser perjudicial ante muestras con alta variabilidad.

Palabras clave: Varianza efectiva, cartas EWMA, proceso multivariado.

\begin{abstract}
This case study presents a monitoring that uses the EWMA control chart based on effective variance, to show how the increase in variability of one quality feature affects the results for this type of chart in a sensitive way. In addition, this allows to expand the capacity to select different monitoring methods for multivariate process. In this research study, subgroups submitted online to a manufacturing process of plant protectants or fungicides were evaluated by using
\end{abstract}

1. Artículo de reporte de caso, tipo de enfoque cuantitativo, resultado de un proyecto de investigación en curso, cartas de control multivariado, perteneciente al área de control estadística de procesos, subárea de estadística industrial, desarrollado en el grupo de investigación Gestión de la Calidad, financiado por la Universidad del Atlántico, de la ciudad de Puerto Colombia (Colombia). Dirección: Calle 30 n. . 8-49, PBX: (57) (5) 3852266. Fecha de inicio: febrero de 2018. Fecha de terminación: febrero de 2019.

2. Ingeniero industrial. Vinculado al grupo de investigación Gestión de la Calidad, de la Universidad del Atlántico, de la ciudad de Puerto Colombia. Dirección: sede centro carrera 43 n. ${ }^{\circ}$ 50-53 Barranquilla (Colombia), PBX: (57) (5) 3852266. ORCID ID: https://orcid.org/0000-0002-8974-1248. Correo electrónico: rogelio.herrera23@hotmail.com

3. Doctorado (c) en la Facultad de Ciencias Económicas y Contables de la Universidad Central de Venezuela. Docente de la Universidad del Atlántico. Barranquilla (Colombia). Vinculado al grupo de investigación Gestión de la Calidad, de la Universidad del Atlántico, de la ciudad de Puerto Colombia. Dirección: sede centro carrera 43 n. ${ }^{\circ}$ 50-53. Barranquilla (Colombia), PBX: (57) (5) 3852266. ORCID ID: https://orcid.org/0000-0003-48554830. Correo electrónico institucional: robertoherrera@mail.uniatlantico.edu.co 
two variables resulting from the process, once the parameters were established. When analyzing the EWMA control chart based on effective variance, it was found that it generates very closed limits, which could be detrimental to samples with high variability.

Keywords: Effective variance, EWMA charts, multivariate process.

\section{Introducción}

Los aspectos que se tienen en cuenta en las cartas de control van cambiando con el paso del tiempo. Tanto así que hace mucho tiempo el aspecto principal a tener en cuenta era la media de los procesos, pero hoy en día no solo se ve la media, sino también la varianza, que se ha ido identificando como una variable fundamental en los diferentes tipos de cartas de control (Koshti, 2013). Ejemplos de estas son Shewart, EWMA y CUSUM. Cada una de estas, con diferentes modelos, han sido presentadas por múltiples autores (Acosta-Mejía, Pignatello \& Reo, 1999; Hawkins, 1981; Hawkins, 1993; Herrera, Mendoza \& Fontalvo, 2016; Karaoglan \& Bayhan, 2012; Montgomery, 1995; Montgomery, 2001).

Uno de los problemas con la varianza ha sido su detección de cambios. En esta se enfocará este estudio. En sus inicios, Peña y Rodríguez (2003) propusieron un modelo que era capaz de hacer, en presencia de conjuntos de variables medidas en las mismas unidades (Alt \& Smith, 1998), comparaciones de conjuntos de estas variables, cuando los conjuntos no tienen igual dimensión. Para ello, utilizaban la varianza efectiva.

Más adelante, Morales y Vargas (2008) realizaron un análisis detallado de las diferentes cartas para cada tipo de varianza global. Estos autores detallaron paso a paso cómo realizar el modelo con carta EWMA para varianza efectiva (Garcias, 2007).

Con base en lo anterior, se desarrolló una aplicación de estas cartas en un caso de estudio real. Este estudio demuestra la funcionalidad de la varianza efectiva.

\section{Marco teórico}

La varianza efectiva propuesta por Peña y Rodríguez (2003) se denota con la siguiente ecuación:

$V E=|\Sigma|^{1 / P}$

Se considera que el conjunto de datos $X$ seleccionados para realizar el control tienen matriz de varianza y covarianza $\bar{S}$. El estadístico $V_{t}$ se define como:

$V_{t}=\frac{\left|S_{t}\right|^{1 / p}}{|\overline{S \mid}|^{1 / p}}$
De acuerdo con la distribución de $V_{t}$, la media y la varianza del estadístico EWMA son:

$S_{v}(t)=\Lambda V_{t}+(1-\Lambda) S_{v}(t-1)$

Están dadas por:

$$
\begin{aligned}
& {\left[E S_{v}(t)\right]=\frac{(n-p)}{(n-1)}\left(\frac{2 n}{2 n-(p-1)(p-2)}\right)^{1 / p}} \\
& \operatorname{Var}\left[S_{v}(t)\right]=\frac{2(n-p)}{p(n-1)^{2}}\left(\frac{2 n}{2 n-(p-1)(p-2)}\right)^{2 / p} \\
& \left(\frac{\Lambda}{2-\Lambda}\left(1-(1-\Lambda)^{2 t}\right)\right)
\end{aligned}
$$

Donde $n$ es el tamaño de la muestra, $p$ es el número de características de calidad o variables del proceso y el vector $\mathrm{X}$ es de dimensión $n \times p$.

De esta manera, los límites de control para la fase II quedan de la siguiente forma (Morales \& Vargas, 2008):

$$
\begin{aligned}
& L C S=\frac{(n-p)}{(n-1)^{2}}\left(\frac{2 n}{2 n-(p-1)(p-2)}\right)^{1 / p}+L \sqrt{\operatorname{Var}\left[S_{v}(t)\right]} \\
& L C=\frac{(n-p)}{(n-1)^{2}}\left(\frac{2 n}{2 n-(p-1)(p-2)}\right)^{1 / p} \\
& L C I=\frac{(n-p)}{(n-1)^{2}}\left(\frac{2 n}{2 n-(p-1)(p-2)}\right)^{1 / p}-L \sqrt{\operatorname{Var}\left[S_{v}(t)\right]}
\end{aligned}
$$

Donde $L$ es una constante, y $\Lambda$ es el parámetro de suavización.

Ahora bien, si se habla de las variables que se analizarán, todas pertenecen a una sustancia conocida como funguicidas. Estas sustancias se utilizan para la protección de cultivos. Es un tipo de plaguicida que busca controlar enfermedades de carácter fúngico, ya sea inhibiendo o eliminando el hongo (FRAC, 2016; McGrath, 2014; Melgarejo \& Abella, 2011).

La mayoría de estos se implementan por medio de fumigación, o se espolvorean sobre las semillas, con el fin de evitar la propagación de los hongos o plagas de estas plantas (Hock, 2012).

El primer fungicida eficaz fue la mixtura de Burdeos, conocida como caldo bordelés, en 1882. Estaba compuesta de cal muerta y sulfato de cobre. Los 
Rogelio José Herrera-Chamorro, Roberto José Herrera-Acosta

Aplicación de la carta EWMA con base en la varianza efectiva para monitorear variabilidad en procesos de control de calidad multivariada de fungicidas

fungicidas pueden ser de carácter preventivo o curativo. Los de prevención buscan evitar que las esporas de los hongos germinen (Martínez \& Guzmán, 2011). Los curativos tienen como objetivo destruir el micelio ya formado (Lyr, Russell \& Sisler, 1996).

Las incidencias por ataques de hongos varían en función de las condiciones ambientales. Dependen de si estos se encuentran a la intemperie o protegidos (Leiva, 2010).

\section{Metodología}

Para este artículo, se escogen como variables los datos de concentración de carbaril (metilcarbamato de 1-naftilo) y el $\mathrm{pH}$ del proceso de fungicidas ilustrado en la Tabla 1. Estas variables se analizan, y entonces se realiza un control de calidad aplicando la carta previamente mencionada. Para ello se escogen cuarenta muestras de una población. Se procede a utilizar las ecuaciones de cartas de control EWMA con varianza efectiva.

Como primera instancia, se procede a centrar los datos. Para esto, se les resta el promedio de la variable menos el promedio de la variable global que fue previamente hallada. Se obtiene así una nueva matriz, como se observa en la Tabla 2 (Herrera, 2006). Luego se determina la matriz de covarianza realizando un producto matricial (esta es la traspuesta de la anterior). Una vez obtenida esta matriz, se toman subgrupos de cuatro datos de cada variable y se determina la matriz de varianza-covarianzas de cada subgrupo.

A partir de las matrices resultantes se calcula el determinante de cada una de ellas. Ya con estos valores se aplica la ecuación (2), y se obtiene $V t$ para cada subgrupo.

Una vez calculado el $S_{v}$ se aplicó la ecuación (3) para hallar el $S_{v}(\mathrm{t})$ de cada subgrupo. Allí $K$ es igual a 0,2 , y se toma como valor de inicio $S_{v(t-1)}=1$.

Luego se aplica la ecuación (5) para hallar la Var $S_{v}(\mathrm{t})$ para cada subgrupo, como se muestra en la Tabla 3. Allí $n$ es el número total de la muestra; y $p$, el número de variables.

Ya con los anteriores datos se pueden hallar los límites de control para la fase II. Esto mediante la utilización de las ecuaciones en (6), respectivamente para cada límite.

Una vez encontrados los límites, se elabora la carta de control EWMA (Gráfica 1), con los datos de los $S_{v}(\mathrm{t})$ Esto para evaluar el proceso, así como para corroborar si se encuentra en control o fuera de control. Entonces se hace el respectivo análisis.

Para evaluar los límites estándar en la fase II de control, se estiman los parámetros $E S_{v}(t)$, con la ecuación (4) y $\operatorname{Var}\left[S_{v}(t)\right]$.

\section{Tabla 1}

Tamaño del subgrupo

\begin{tabular}{|c|c|c|}
\hline & Concentración de Carbaril & pH \\
\hline \multirow{4}{*}{1} & 59,51 & 14,79 \\
\hline & 58,01 & 14,64 \\
\hline & 59,00 & 14,73 \\
\hline & 60,17 & 14,16 \\
\hline \multirow{4}{*}{2} & 61,50 & 14,97 \\
\hline & 61,02 & 14,63 \\
\hline & 59,63 & 15,66 \\
\hline & 60,24 & 14,03 \\
\hline \multirow{4}{*}{3} & 58,95 & 15,03 \\
\hline & 57,98 & 14,34 \\
\hline & 60,44 & 14,70 \\
\hline & 58,91 & 14,19 \\
\hline \multirow{4}{*}{4} & 59,18 & 15,03 \\
\hline & 59,46 & 14,64 \\
\hline & 59,42 & 14,64 \\
\hline & 57,75 & 14,33 \\
\hline \multirow{4}{*}{5} & 59,75 & 15,44 \\
\hline & 58,25 & 14,48 \\
\hline & 59,25 & 14,64 \\
\hline & 59,54 & 14,00 \\
\hline \multirow{4}{*}{6} & 57,69 & 14,52 \\
\hline & 57,63 & 14,40 \\
\hline & 57,26 & 14,64 \\
\hline & 59,94 & 14,57 \\
\hline \multirow{4}{*}{7} & 59,51 & 13,55 \\
\hline & 57,72 & 14,93 \\
\hline & 57,56 & 15,74 \\
\hline & 59,31 & 15,08 \\
\hline
\end{tabular}




\begin{tabular}{ccc} 
& 58,65 & 14,85 \\
8 & 58,64 & 14,78 \\
& 58,83 & 14,55 \\
& 59,87 & 15,15 \\
\hline & 60,05 & 15,09 \\
& 60,30 & 14,93 \\
& 60,99 & 14,13 \\
& 58,22 & 13,97 \\
\hline \multirow{3}{*}{10} & 59,72 & 14,10 \\
& 60,51 & 14,90 \\
& 61,04 & 15,42 \\
& 60,12 & 14,57 \\
\hline
\end{tabular}

Fuente: Autores.

\section{Tabla 2}

Concentramiento de la información

\begin{tabular}{ccc}
\hline & $\begin{array}{c}\text { Datos Centrados } \\
\text { Concentración }\end{array}$ & $\begin{array}{c}\text { Datos } \\
\text { Centrados } \mathbf{~ H}\end{array}$ \\
\hline & 0,22 & 0,12 \\
& $-1,28$ & $-0,03$ \\
& $-0,29$ & 0,06 \\
& 0,88 & $-0,51$ \\
\hline & 2,21 & 0,30 \\
& 1,73 & $-0,05$ \\
& 0,34 & 0,99 \\
3 & 0,95 & $-0,65$ \\
\hline & $-0,34$ & 0,36 \\
& $-1,31$ & $-0,33$ \\
& 1,15 & 0,03 \\
& $-0,38$ & $-0,48$ \\
\hline \multirow{3}{*}{4} & $-0,11$ & 0,36 \\
& 0,17 & $-0,03$ \\
& 0,13 & $-0,03$ \\
& $-1,54$ & $-0,35$ \\
\hline \multirow{3}{*}{5} & 0,46 & 0,76 \\
& $-1,04$ & $-0,20$ \\
& &
\end{tabular}

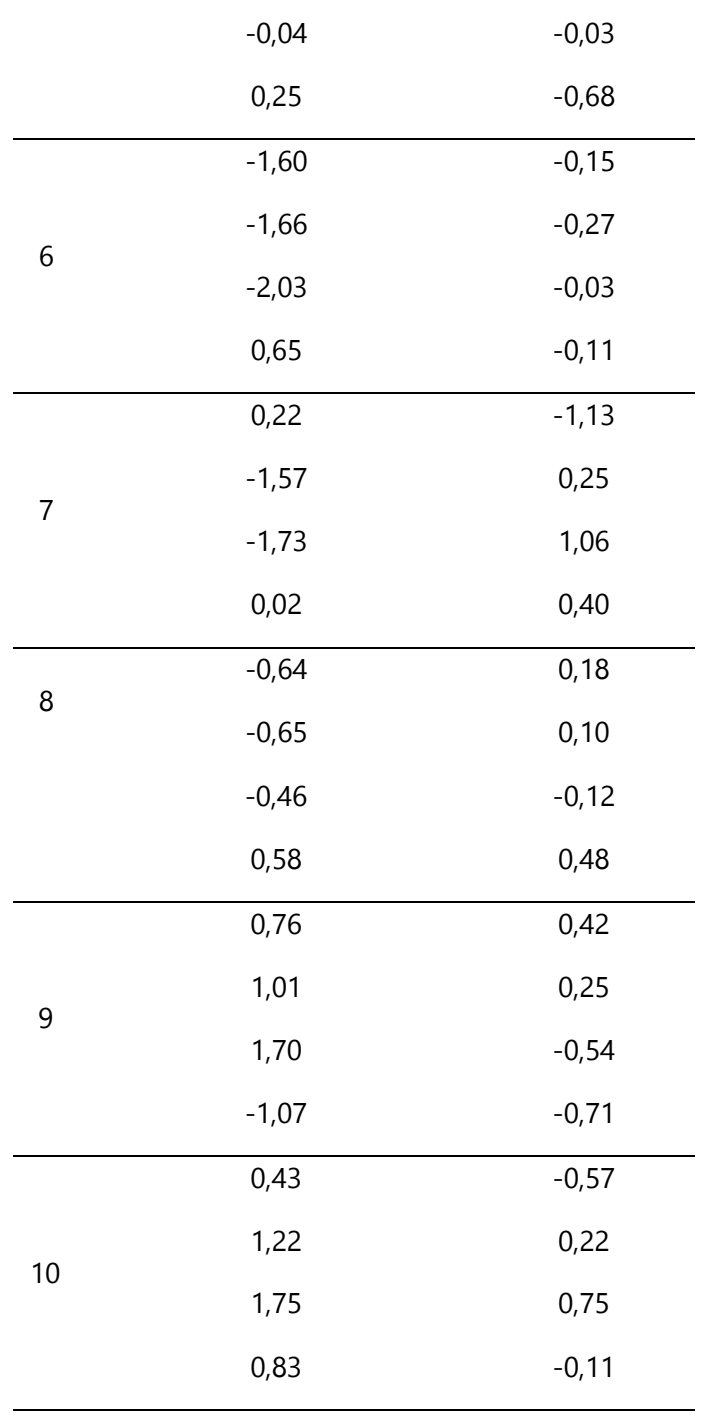

Fuente: Autores.

\section{Resultados}

Al utilizar cada una de las ecuaciones con los datos obtenidos en las muestras, se obtienen los siguientes parámetros y variables. Estos son pertinentes para la elaboración de nuestra carta de control.

La matriz de varianza covarianza se conoce como:

$$
\Sigma=\left[\begin{array}{cc}
0,063 & -0,287 \\
-0,287 & 1,634
\end{array}\right]
$$

\section{Tabla 3}

Matriz de datos obtenidos por subgrupo

\begin{tabular}{ccc}
\hline Subgrupo & $\mathbf{v}_{\mathbf{t}}$ & $\mathbf{S v}_{\mathbf{t}}$ \\
\hline 1 & 0,396 & 0,879
\end{tabular}




\begin{tabular}{|c|c|c|}
\hline 2 & 1,934 & 1,090 \\
\hline 3 & 0,602 & 0,992 \\
\hline 4 & 0,325 & 0,859 \\
\hline 5 & 0,611 & 0,809 \\
\hline 6 & 0,413 & 0,730 \\
\hline 7 & 1,536 & 0,891 \\
\hline 8 & 0,324 & 0,778 \\
\hline 9 & 1,267 & 0,876 \\
\hline 10 & 1,011 & 0,903 \\
\hline
\end{tabular}

Fuente: Autores.

La esperanza de los datos hallada con (4) es 0,75; y la varianza, 0,109, para un tamaño de subgrupo de cuatro $n=4$ y dos variables $p=2$. Sus límites superior, central e inferior, mediante la ecuación (6), son 1,74, 0,75 y -0,24, respectivamente.

Con todos los valores y parámetros previamente hallados, se construye la Gráfica 1 para la carta de control EWMA. Así se procede a analizar el comportamiento de las variables.

\section{Análisis de resultados}

Después de realizar los pasos para obtener los límites de control estándar, se encontró que estos valores son pequeños. Sin embargo, los datos sometidos a control se encuentran dentro de los límites establecidos. Los subgrupos seleccionados arrojan varianzas efectivas de las variables del proceso, como se aprecia en la Tabla 3. Estos valores están sujetos al número de observaciones seleccionadas en la muestra.

Como se puede observar en la Gráfica 2, en la que se evalúa a partir de los parámetros respectivos, la mayoría de los puntos sometidos a control se acercan al límite de control inferior. Este comportamiento sugiere que la medida de variabilidad conjunta disminuyó considerablemente, con respecto al comportamiento histórico. Es decir, el proceso a corto plazo o control presenta una disminución de la variabilidad. Es recomendable, en este sentido, realizar una revisión del proceso, debido a una posible falsa alarma, o, en el mejor de los casos, un estado que indique un mejoramiento en el comportamiento del proceso.

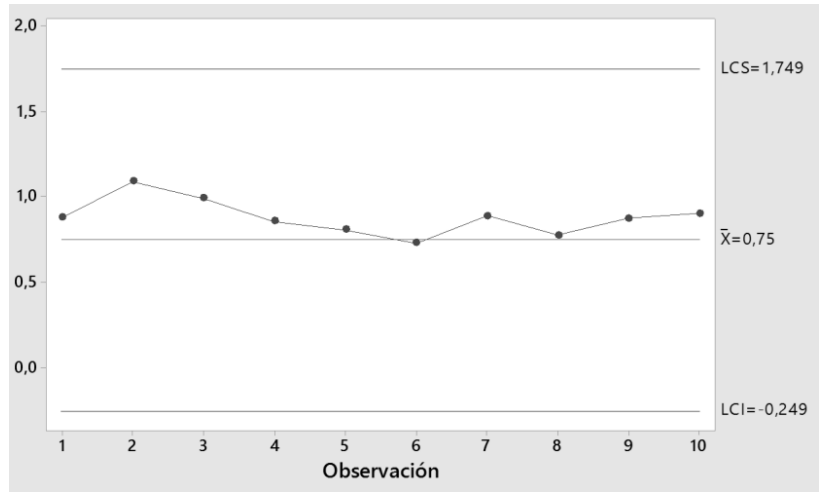

Gráfica 1. Carta de control EWMA con varianza efectiva, con los datos de $\mathrm{pH}$ y concentración de carbaril (metilcarbamato de 1-naftilo) de funguicidas. Fuente: Autores.

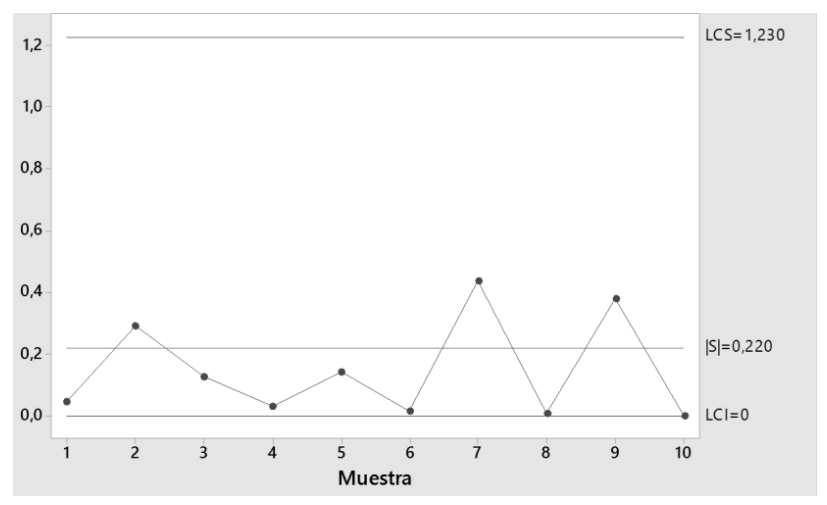

Gráfica 2. Carta de control varianza generalizada con los datos de $\mathrm{pH}$ y concentración de funguicidas. Fuente: Autores.

\section{Conclusión}

En la utilización de los funguicidas, la calidad del producto empleado es de alta consideración. Esto se debe a que el implementar una sustancia que posea niveles de $\mathrm{pH}$ o concentración de carbaril (metilcarbamato de 1-naftilo) por encima de lo permisible llevaría a afectar el cultivo, y esto representaría pérdidas para los agricultores. Si, por lo contrario, están por debajo, esto puede no afectar al hongo o plaga que se desea atacar, lo que genera las mismas consecuencias.

En el análisis realizado de la calidad del funguicida, se implementó la carta EWMA con varianza efectiva propuesta por Morales y Vargas (2008). Como resultado se obtuvo una carta de control con límites muy cerrados, lo que podría ser perjudicial ante muestras con alta variabilidad. En dicho caso, no es recomendable la 
Rogelio José Herrera-Chamorro, Roberto José Herrera-Acosta Aplicación de la carta EWMA con base en la varianza efectiva para monitorear variabilidad en procesos de control de calidad multivariada de

implementación de esta propuesta en procesos con una dispersión considerable. Sin embargo, es indispensable recalcar que existen procesos químicos que requieren un monitoreo estricto por las condiciones fisicoquímicas de la característica de calidad. En el caso de la concentración de carbaril, se recomienda la implementación de este tipo de carta en el análisis de datos de procesos sensibles a la salud humana.

\section{Bibliografía}

Acosta-Mejía, C., Pignatello, J., \& Reo, V. (1999). A comparation of control charting procedures for monitoring process dispersion. IIE Transactions, 31(6), 569-579.

Alt, F.B., \& Smith, N.D. (1998). Multivariate Process Control. Handbook of Statistics 7, 333-351.

FRAC (2016) (Fungicide resistance action committee), Clasificación defungicidas. Recuperadodehttps://www. queaplico.com/biblioteca?id=7fungicidesSpanish.aspx

Garcias, J. C. (2007), The Effective Variance Control Chart for Monitoring the Dispersion process with Missing data, European J. Industrial Engineering, 1, 40-55.

Hawkins, D.M, (1981). A CUSUM for a scale parameter. Journal of Quality Technology, 13(4), 228-231.

Hawkins, D.M, (1993). Cumulative Sum Control Charting: An Underutilized SPC Tool. Quality Engineering, 15(3), 463-477.

Herrera, R. J., Mendoza, A., \& Fontalvo, T. (2016). Comparación de las Cartas de Control Univariadas con Transformación en la Medida de Variabilidad. Ingeniare, (13), 51-57.

Herrera, R. J. (2006). Seis Sigma Métodos Estadísticos y sus Aplicaciones. Ecoe Ediciones, pp. 56.

Hock, W. K. (2012) "Effect of pH on pesticide stability and efficacy," Pesticide Safety Education Program (PSEP). Cornell University. Recuperado de http:// psep.cce.cornell.edu/facts-slides-self/facts/genpeapp-ph.aspx.

Karaoglan, A. D., \& Bayhan, G. M. (2012). ARL performance of residual control charts for trend AR (1) process: $A$ case study on peroxide values of stored vegetable oil. Scientific Research and Essays, 7(13), 1405-1414.

Koshti, V. V (2013). Cumulative sum control chart. International Journal of Physics and Mathematical Sciences, 1(1), 28-32.

Leiva P. D. (2010). Consideraciones generales sobre calidad del agua para pulverización agrícola. INTA. Argentina

Lyr, H., Russell, P. E., \& Sisler, H. D. (1996). Modern Fungicides and Antifungal Compounds. Andover, United Kingdom.: Intercept Limited.

Martínez, I., \& Guzmán, M. (2011). Guía básica para la preparación de mezclas, uso de fungicidas y calibración de motobombas utilizadas en el combate de la Sigatoka negra. Oeste, 300(1).

McGrath, M.T. (2014) "¿Qué son los fungicidas?" The Plant Health Instructor. Trans. L. Santamaría, J.C Ureta, Cornell University. Recuperado de http:// www.apsnet.org/EDCENTER/INTROPP/TOPICS/ Pages/

Melgarejo, J., \& Abella, F. (2011). Mecanismo de acción de los fungicidas. Revista Ventana Al Campo, 193202.

Montgomery, D. C (2001). Introduction to statistical Quality Control. Wiley, New York, United States.

Montgomery, D.C. (1995). Control estadístico de la calidad. Limusa, 3a Edición, ciudad de México, México, pp. 406-424.

Morales, V. H. \& Vargas J. A. (2008), Carta EWMA con varianza efectiva para monitorear variabilidad en procesos de control de calidad multivariado. Colombia.

Peña, D. \& Rodriguez J. (2003), Descriptive Measures of Multivariate Scatter and Linear Dependence, Journal of Multivariate Analysis, 85, 36-374.

Wilks, S. S. (1932), Certain Genereralizations in the Analysis of Variance, Biometrika, 24, 471-494 